\title{
Flexible Unmanned Surface Vehicles enabling Future Internet Experimentally-driven Research
}

\author{
Bruno Ferreira, André Coelho, Mário Lopes, Aníbal Matos, Carlos Gonçalves, \\ Saravanan Kandasamy, Rui Campos, Jorge Barbosa \\ INESC TEC and Faculdade de Engenharia, Universidade do Porto \\ Rua Dr. Roberto Frias, 4200-465 Porto, Portugal \\ Email: \{bmmf,afcoelho,mjlopes,anibal.matos,carlos.f.goncalves,kandasamy,rcampos,jorge.f.barbosa\}@inesctec.pt
}

\begin{abstract}
FLEXUS unmanned surface vehicle was designed in the context of the Internet of Moving Things. This small catamaran weights less than $15 \mathrm{~kg}$ and is less than $1 \mathrm{~m}$ long, making it a very convenient vehicle with reduced logistics needs for operations in real outdoor environments. The present paper describes the resulting system both in terms of design and performances. Based on the requirements for this project, the subsystems composing the vehicle are described. Results obtained from experiments conducted in outdoor conditions have successfully validated this design and are presented in this paper.
\end{abstract}

\section{INTRODUCTION}

This paper describes FLEXUS - a large fleet of small size and affordable unmanned surface vehicles (USVs) developed for field experiments within the scope of the Road-, Air-, and Water- based Future Internet Experimentation (RAWFIE). This European project aims at providing tools for remote experimentation of multiple robotic platforms over the Internet. When dealing with several USVs operating simultaneously, it is of utmost importance to guarantee safety, ease of deployment and handling. In the context of logistics, the USVs should be small in dimensions and weight but their capability of carrying a small amount of payload should not be neglected. To guarantee safety and coherency on the fleet's motion, the USVs should be able to wirelessly communicate and coordinate to achieve a mission's objectives without human intervention once the vehicles are powered on and commanded to start the mission. FLEXUS USVs are the result of a careful design, balancing performance, communications advancement, additional payload capacity, dimensions and weight. These USVs are aimed at enabling a large number of experiments within the context of the Internet of Moving Things at sea, being capable to execute a basic and yet powerful set of actions. In this context, communications between the USVs and a central entity are of crucial importance. The USVs are equipped with wireless communications offering two redundant interfaces to communicate with a base station and an additional interface functioning as an Access Point enabling the attachment of legacy devices to communicate via the USVs.

\section{A. Related works}

Some companies have proposed small, lightweight USVs such as the two-men-portable Martac Mantas [1], Evologics Sonobot [2], Sonarbeam SB-100 [3], Clearpath Heron [4] and Deep Oceans Phantom I-980 [5]. The applications that these vehicles are able to cover is wide, ranging from surveillance to scientific data collection. Apart from Mantas, whose characteristics are customizable, the remaining USVs are more than 1-meter long and weight some tens of kilograms, thus making them convenient for constrained logistics applications. Concerning small size vehicles, we should also include the Portuguese semi-submersible Medusa [6], developed by IST, and the ISCTE prototypes [7]. Despite their interesting characteristics in terms of dimensions and weight in the context of RAWFIE, their capabilities to accommodate additional payload are strongly limited.

Several robotic surface platforms have been designed and developed at INESC TEC for the last decade. The concerns related to logistics and ease of deployment gave raise to smallsized, lightweight USVs serving several scientific research purposes, in which their payload capabilities (above 10kg), play an important role. Zarco [8] and the Unmanned Capsule

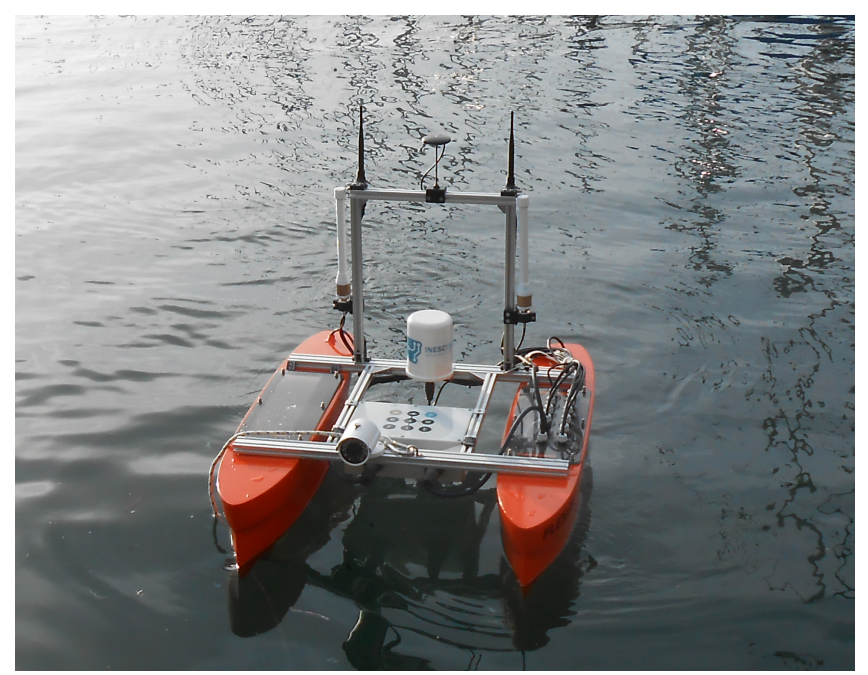

Fig. 1: FLEXUS USV 
(UCAP) [9] are two examples of such a type of vehicles. Although relatively small and lightweight compared to most USVs solutions, these vehicles require two operators to deploy them.

Regarding above water maritime wireless communications, the works in the literature to provide cost-effective and high bit rate solutions so far have been based on WiMAX technology. Some examples are the buoy-to-ship point-to-point communications solution using the $5.8 \mathrm{GHz}$ band [10], the performance evaluation of ship-to-shore point-to-point communications [11], and the multi-hop ad-hoc routing protocol and cognitive medium access control mechanism for long range ship-to-shore communications [12]. Though WiMAX based solution is good, the capital expenditure (CAPEX) for WiMAX is high. In FLEXUS, Wi-Fi based solution is explored where it has a lower CAPEX value than WiMAX making the solution more affordable.

The Mare-Fi project is a research and development effort enabling affordable Internet access for fishing industry using Wi-Fi-based high bitrate and long range maritime communications [13]. The experiments carried out in Mare-Fi allowed the authors to provide ship-to-shore broadband IP-based communications up to $10 \mathrm{~km}$ from shore, however the backbone communication is limited to single-hop nodes [14] [15] [16]. In FLEXUS, multi-hop communication using Wi-Fi is explored.

\section{B. Organization}

This paper is organized as follows: section II lists the main requirements for the FLEXUS USVs, which establish the baseline for the vehicle design, then described in section III. Section IV presents the results from a set of experiments conducted in a typical outdoor environment. Final remarks are presented in section $\mathrm{V}$.

\section{REQUIREMENTS}

Operating several vehicles simultaneously may lead to significant logistics and setup efforts, often requiring logistics assistants and/or several operators. Reducing dimensions and weights considerably helps on the logistics, while auto-configuration positively impacts on setup efforts. The FLEXUS USVs are targeted at scientific applications demanding efficient motion with reduced logistics needs and fast deployment. Their requirements were split into four main categories: i) mechanical; ii) sensing and software; iii) communications; iv) operational.

Below, the main requirements are presented.

- Mechanical

- A single person should be able to handle the vehicle alone. The USV dimensions should not exceed $1.0 \times 1.0 \times 1.0 \mathrm{~m}$ and the weight must not exceed $18 \mathrm{~kg}$.

- The USV must be capable of carrying external equipment up to a volume of $0.4 \times 0.4 \times 0.2 \mathrm{~m}$ and weighing up to $5 \mathrm{~kg}$.

- Sensing and software

- The USV must be able to sense their positions.
- The vehicle must be able to determine their attitude.

- The USV must interface with the RAWFIE back-end for sending telemetry and sensed data, and receiving commands.

- Communications

- The vehicles must be able to operate in two wireless frequency bands $(2.4 \mathrm{GHz} / 5 \mathrm{GHz})$ simultaneously.

- Access Point functionality must be implemented on each vehicle.

- Operational

- The USV must have two degrees of freedom

- The USV maximum speed must be at least 2 knots

- The vehicles must be able to perform missions composed of waypoints and station keeping.

- Their minimum autonomy must be at least 1 hour at 2 knots.

\section{USV DESIGN}

The vehicle design was the result of previous implementations and experience over several projects and vehicles. As such, the design relied on as much consolidated technology as possible. The main efforts were focused on the body, the payload configurations and on the telecommunications to enable easy handling and deployment, versatility and link reliability over extended areas.

\section{A. Body}

Based on INESC TEC's experience in the field, weight and dimensions are features that matter for logistics reasons. Small vehicles naturally have the advantage of less demanding requirements for transportation, deployment and recovery.

Bearing in mind logistics, ability to operate in indoor and outdoor environments, ease of deployment and recovery, the USV was designed to take the form of a small-size catamaran (see Fig.1) with less than one meter in length, weighting about $15 \mathrm{~kg}$ and yet capable to easily accommodate additional payload (about $5 \mathrm{~kg}$ ).

The body is composed of two parallel fiberglass floaters jointly designed by INESC TEC and Arsenal do Alfeite (Fig. 1-2). Their shape has been designed to guarantee efficient motion of the USV, and for providing buoyancy to the USV and to a possible extra payload. The resulting design led to a $90 \mathrm{~cm}$ long by $17 \mathrm{~cm}$ wide floater, with a height of $20 \mathrm{~cm}$. Embedded screws and inserts were added to the floaters to enable fastening of coupling structures, actuators and waterproofing hatches.

Additionally, their hollow nature has been used for accommodating the vehicle's equipment, thus minimizing the amount of devices outside the floaters, resulting in a compact solution.

A structured aluminum frame links the two floaters. Beyond the structural strength provided to the USV, this frame also creates a deck surface for external equipment and payload. A battery pack is currently attached to this structure, which is a natural choice for appending external sensors. A bridge was mounted on the deck to support communication and GPS antennas, elevating them from the water surface. 


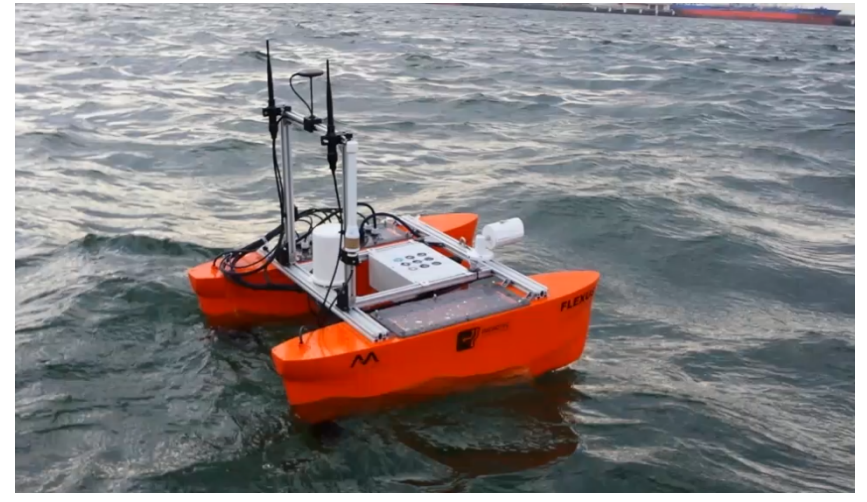

Fig. 2: FLEXUS USV navigating

\section{B. Electronics}

FLEXUS USVs implement a core set of devices that enable autonomous navigation. The main blocks are:

- Battery pack

- Computational system

- Wireless communication system

- Actuation pack

- Global Positioning System (GPS)

- Attitude sensor

1) Battery pack: The battery pack is composed of four $\mathrm{LiFePO} 4$ batteries connected in series providing a nominal capacity of $240 \mathrm{Wh}$ at $13.2 \mathrm{~V}$. A battery monitoring system was developed to assess the battery health and to protect it against overcurrent and overdischarge, which is critical for this type of batteries. Beyond monitoring tasks, the system integrates an USB connection over which it is able to provide information on battery status, namely drawn current, voltage and remaining charge.

It was important in the vehicle design to enable detaching the battery pack not only because of transportation concerns but also for ease of handling for charging.

2) Computational system: The computational system of FLEXUS is composed of a single board computer equipped with a dual core ARM11 SoC processor operating at $600 \mathrm{MHz}$, 256Mbytes of DRAM memory and 16Mbytes of system flash. The single board computer includes four high-power miniPCI sockets, which enable the combination of four wireless Network Interface Controllers (NICs), and two Ethernet ports. A micro SD flash socket, and two USB ports for expansion are also embedded on the single board computer [17].

The operating system for the single board computer runs on a customized OpenWrt Bleeding Edge (trunk version) image. OpenWrt is an open-source and community-driven Linuxbased distribution for embedded systems, primarily designed to enhance the integration of network components on devices with limited storage, reduced memory resources, and lowpower consumption [18].

3) Wireless communication system: The wireless communication system on each USV is formed by three wireless NICs. Two of them are operating at the $2.4 \mathrm{GHz}$ and $5 \mathrm{GHz}$
ISM bands, respectively, forming a Core Network; the third wireless NIC aims to provide a standard Access Network at $2.4 \mathrm{GHz}$ ISM band, enabling the connection of legacy WiFi devices. Both Core Network's wireless NICs are attached to the computational system's mini-PCI sockets. The Access Network's wireless NIC is plugged via USB, in order to allow its positioning farther away from the remaining NICs, aiming to minimize self-interference between NICs. For the Core Network four omnidirectional antennas are being used, two for each frequency band, in order to explore MIMO techniques. For the Access Network, a single omnidirectional antenna, radiating upwards, is being used.

4) Actuation pack: The actuation block is composed of three main modules: thrusters, electronic speed controllers and an interface board. Two thrusters in a differential configuration are used to control forward force and yaw (rotation about vertical axis) moment. With this configurations, the vehicle is virtually capable to control surge and yaw independently. These are placed at stern by means of two screws and their respective inserts embedded in the floaters hulls, enabling easy and fast replacement of thrusters. The electronic speed controllers are standard devices commanded by a pulse-width modulated signal, which is generated by the actuation interface electronic board. This board offers a USB connection from which it receives commands for the two thrusters and returns back data on voltage and current drawn by the two motors independently.

5) Global positioning system and attitude sensor: Standard GPS receiver and a magnetometer were integrated in FLEXUS for pose determination. These devices generate data streams over universal asynchronous receiver/transmitter (UART) and I2C, respectively. In order to centralize and to reduce the number of interface devices, a data centralizer based on a microprocessor was developed to conduct the data to the onboard PC.

\section{Communications}

In the maritime environment, narrowband wireless communications are still the norm using HF/VHF analog radios. Broadband wireless communications are limited to near-shore zones using cellular networks ( $3 \mathrm{G} / 4 \mathrm{G}$ ) or can only be achieved using satellite communications that are still too expensive for most of the maritime users and still support low bit rates.

FLEXUS USVs support affordable, broadband wireless communications, paving the way to experimentally-driven research on Future Internet in maritime environment, namely offshore, thanks to the use of long-range and multi-hop communications. FLEXUS USVs are equipped with multiple WiFi interfaces, using the $2.4 \mathrm{GHz}$ and $5 \mathrm{GHz}$ bands, which are used to enable the attachment of legacy Wi-Fi devices. Multihop communications among FLEXUS USVs are enabled by means of any routing protocol, either those already available and running under Linux / OpenWRT or any new developed routing protocol that can run under such operating systems.

With FLEXUS USVs it is possible to run experiments up to several kilometers from shore or from a mission- 
support ship, in contrast to current plain Wi-Fi enabled USVs, which are limited to a few hundred meters range. With these features FLEXUS USVs are at the forefront in enabling experimentally-driven research on the Internet of Moving Things at sea.

\section{Configurations}

FLEXUS USVs are intended to cover a broad range of data sampling applications. As such, versatility is desired and it is ensured by easy payload reconfigurations. Three sensor packs are currently envisaged:

- Imaging (HD camera)

- Water monitoring (temperature, $\mathrm{pH}$, conductivity)

- Air monitoring (temperature, $\mathrm{CO}, \mathrm{CO} 2$ )

It should be noted that the payload is not limited to these sensors and can be extended and adapted depending on the application.

\section{EXPERIMENTAL RESULTS}

Experiments were designed to validate the subsystems and assess their performance. Before first deployment, the subsystems were evaluated both in terms of performances and stability over extended periods of operation. The validation experiments included thruster operation under different actuation conditions, wireless communications bandwidth and range, GPS and magnetometer stability, computer and operative system stability under typical operational conditions.

After validation, achieved by analyzing several hours of $\operatorname{logs}$, the vehicle was prepared for the first validation missions. The first validation experiments were programmed for basic testing of the vehicle in outdoor, challenging conditions. An operator was continuously remotely operating the USV over the entire operation, which took place in a harbor near Porto, Portugal. The main objective of this mission was to validate the complete solution in real conditions, to collect data and optimize minor variables such as weight distribution, communication-related parameters and control software parameters.

In a typical operation, the minimal setup is composed of the vehicle and a base-station that serves as a gateway to the vehicle. Additionally, it also serves as a central point, possibly with an Internet connection, enabling remote mission programming and real-time feedback to a remote operator.

\section{A. Field trials}

The first field trials of the final system solution were carried out near Lisbon, in the Tejo river, basing the operation in the Portuguese Naval base in Almada. The main objectives of these trials were:

- Validate the USV solution

- Identify possible issues

- Test remote command

- Test autonomous maneuvers

- Evaluate performances

- Maximum velocity

- Autonomy
- Evaluate the telecommunications solution

- Maximum range

- Throughput vs distance

A set of intensive experiments extending over two days were designed. Two battery packs were used to allow continuous operation of the USV. While the vehicle was operating with one battery pack, the remaining battery pack was put to charge.

The experiments included both remote commands, assuming an operator, and autonomous missions. Remote command included direct commands to the thrusters and target tracking/station-keeping (set by the operator) maneuvers. Autonomous missions included waypoint tracking and stationkeeping instructions. An example of a "sweeping" mission, composed of a set of waypoints, is presented in Fig. 3. This figure illustrates the vehicle capability to follow lines connecting consecutive waypoints. The mission was designed for the vehicle to follow $100 \mathrm{~m}$ long parallel legs, $10 \mathrm{~m}$ apart from each other. The figure demonstrate that the FLEXUS USV was able to track the lines with very satisfactory performances. The cross-track error was typically well under $1 \mathrm{~m}$ after stabilizing.

The station-keeping maneuver was also validated over a remotely set instruction. A point was arbitrarily chosen on a map of the operation console and sent over $\mathrm{Wi}-\mathrm{Fi}$, which also support data telemetry and control data exchange. The result of the station-keeping maneuver is shown in Fig. 4. As depicted from the position graphs (North and East), the vehicle was able to stabilize and keep its final position with a variance of less than one meter. The heading graph shows the vehicle's effort to face the disturbances in order to hold the position.

In both waypoint tracking and station-keeping operations, it should be noted that the position data is obtained directly from the GPS, used to feed back controllers in real-time. No other ground-truth method was applied. The results are naturally corrupted by the error of the device, which has a standard accuracy of $2 \mathrm{~m}$, but the output data shows the vehicle capability to track lines and to hold its position precisely. Furthermore, during the entire operation, the USV was subjected to disturbances caused by wind, currents and waves, whose influences could not be quantified but have an obvious impact on the vehicle.

During these field trials, the vehicle covered more than $23 \mathrm{~km}$ of distance, $8.3 \mathrm{~km}$ of which were covered on a single battery pack charge.

A set of experiments were also performed in order to assess and validate the communications solution. Iperf tool was used to generate UDP traffic with a bitrate of $100 \mathrm{Mbit} / \mathrm{s}$, during 180s duration per experiment. Additionally, in order to measure the Round-Trip-Time (RTT), after each Iperf test a ping test of 10 s was performed.

The experimental results were obtained considering two single-hop wireless links, each operating in $2.4 \mathrm{GHz}$ and $5 \mathrm{GHz}$ frequency bands respectively, between base-station and USV using the IEEE 802.11n standard. The transmission power was set to $20 \mathrm{dBm}$ for $2.4 \mathrm{GHz}$ with $4 \mathrm{dBi}$ gain single-band antennas, and to $15 \mathrm{dBm}$ for $5 \mathrm{GHz}$ with $3 \mathrm{dBi}$ dual-band antennas. Both nodes have been configured in Ad-hoc mode 


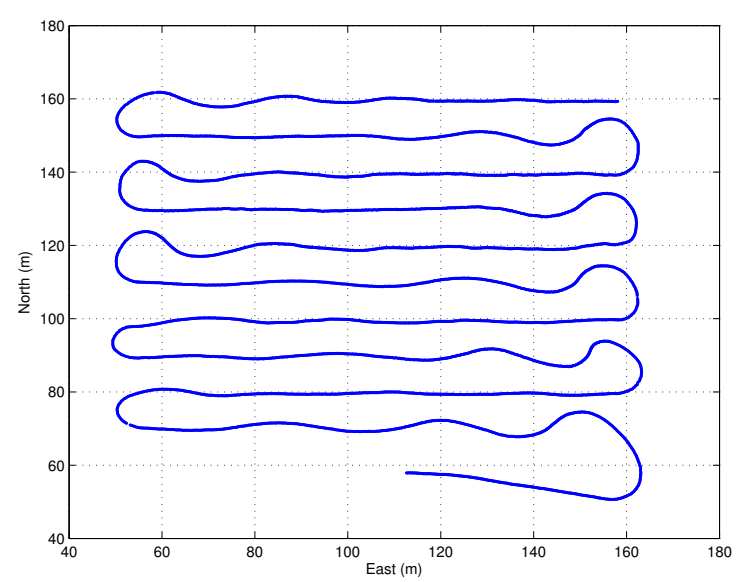

Fig. 3: Vehicle trajectory in a typical sweeping mission
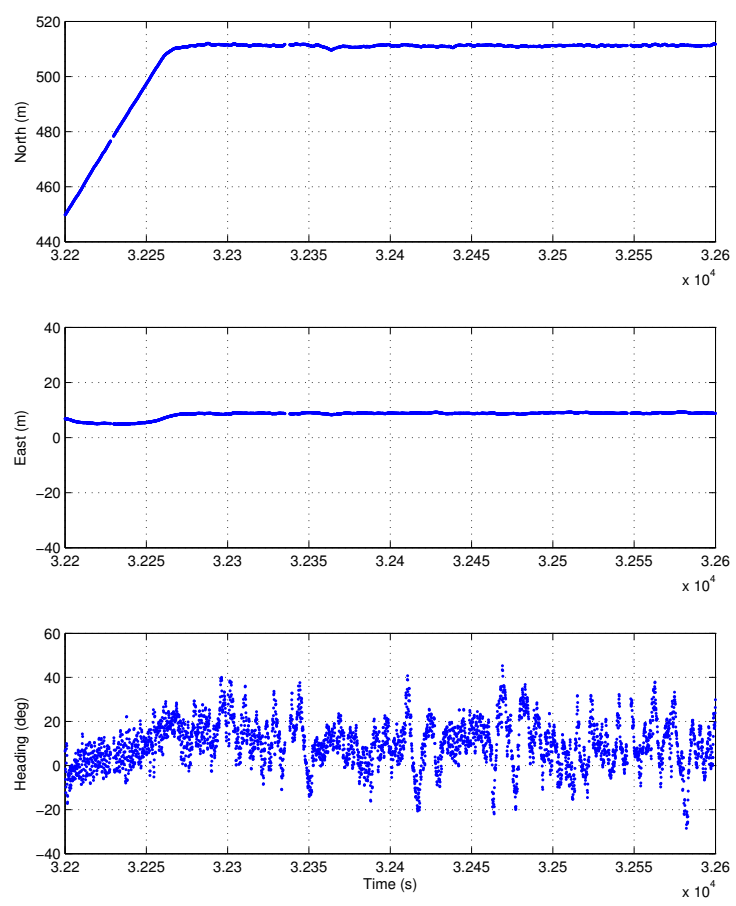

Fig. 4: Station keeping results

running on the B.A.T.M.A.N. (Better Approach To Mobile Ad-hoc Networking) routing protocol, due to its i) superior performance on networks with unreliable links and ii) lower control overhead [19]. The third wireless NIC, which is intended to provide an Access Network in the USV, was not used to exchange traffic during the experiments, however it was turned on to allow beacon frames to be transmitted periodically to the air.

Table I shows the UDP throughput and ping RTT values obtained for reference purposes, considering $20 \mathrm{~m}$ distance
TABLE I: Reference UDP throughput and ping RTT obtained for IEEE $802.11 \mathrm{n}$-like $2.4 \mathrm{GHz}$ and $5 \mathrm{GHz}$ links between both base-station and USV for a range of $20 \mathrm{~m}$ (LOS).

\begin{tabular}{|c|c|c|c|}
\hline Scenario & $\begin{array}{c}\text { Frequency } \\
\text { (GHz) }\end{array}$ & $\begin{array}{c}\text { UDP Throughput } \\
\text { (Mbit/s) }\end{array}$ & $\begin{array}{c}\text { Ping RTT } \\
\text { (ms) }\end{array}$ \\
\hline \multirow{2}{*}{ Single link } & 2.4 & 25.20 & 2.1 \\
\cline { 2 - 4 } & 5 & 40.20 & 2.1 \\
\hline \multirow{2}{*}{ Dual link } & 2.4 & 14.14 & 6.8 \\
\cline { 2 - 4 } & 5 & 31.60 & 2.4 \\
\hline
\end{tabular}

TABLE II: UDP throughput and ping RTT obtained for IEEE 802.11 n-like $2.4 \mathrm{GHz}$ and $5 \mathrm{GHz}$ links between both basestation and USV for a range of $550 \mathrm{~m}$ (LOS).

\begin{tabular}{|c|c|c|c|}
\hline Scenario & $\begin{array}{c}\text { Frequency } \\
(\mathbf{G H z})\end{array}$ & $\begin{array}{c}\text { UDP Throughput } \\
(\mathbf{M b i t} / \mathbf{s})\end{array}$ & $\begin{array}{c}\text { Ping RTT } \\
(\mathbf{m s})\end{array}$ \\
\hline \multirow{2}{*}{ Single link } & 2.4 & 0.3350 & 318.7 \\
\cline { 2 - 4 } & 5 & 4.62000 & 5.9 \\
\hline \multirow{2}{*}{ Dual link } & 2.4 & 0,02356 & 1931.7 \\
\cline { 2 - 4 } & 5 & 0,05876 & 2.6 \\
\hline
\end{tabular}

link between both nodes, which were in Line-Of-Sight (LOS). Table II shows the UDP throughput, as well as the RTT values for $550 \mathrm{~m}$ distance link; this was the maximum distance for which a full control of the USV and bidirectional data exchange with the base-station was achieved, considering a LOS scenario. The results of the experiments presented in both tables are divided by scenarios in order to characterize the communications solution either when traffic flows through a single link, and using both links simultaneously.

From the communications point of view, the field trials allowed to validate the range of the FLEXUS solution for a distance up to $550 \mathrm{~m}$, with throughput values compatible with the intended type of application. Yet, note that this range will be higher (up to several kilometers) considering the complete FLEXUS fleet, since the number of hops will be increased. Regarding the lower throughput values when traffic flows through both links simultaneously (dual link scenarios in both Table I and Table II), this phenomenon can be explained due to eventual self-interference issues between the Core Network's wireless NICs, however this will not be a problem in the FLEXUS final solution, since the two links will assume a redundant role and only in exceptional scenarios will be used simultaneously to exchange traffic. The final version of the telecommunications solution will allow running experiments, triggered anywhere over the Internet, up to several kilometers from shore or from a mission-support ship, in contrast to current plain Wi-Fi enabled USVs, which are limited to a few hundred meters range. Considering all the features together, we argue that FLEXUS solution is placed at the forefront on experimentally-driven research on the Internet of Moving Things and will generate the potential to attract experimenters from all over the world.

\section{CONCLUSIONS AND FUTURE WORK}

This paper presented the design and the validation of the FLEXUS USV. The first prototype was conceived to test and evaluate the FLEXUS concept. The experimental results have 
demonstrated outperforming navigation capabilities, autonomy and ability of vehicles to communicate distance up to $550 \mathrm{~m}$ point-to-point.

The final version of this prototype will implement several envisioned improvements, including lighter structure and battery pack allocation. From the telecommunications point of view, some tests are being planned in order to fully characterize the performance of the FLEXUS solution in a multi-hop sea testbed formed by a fleet of USVs, aiming to obtain quantitative and qualitative contributions, including the behavior of the B.A.T.M.A.N. protocol, radio coverage, throughput values, and relevant signal propagation issues. This will represent a step forward to close the gap on performance characterization of wireless vehicular networks in above water scenarios.

\section{ACKNOWLEDGEMENT}

The authors thank Arsenal do Alfeite for the contributions on mechanical design, construction of the floaters and for providing means to conduct the experiments referred in this paper. Our special thanks go to Mário Figueiredo and Fernando Cruz for their support in all these phases and for sharing their meaningful experience throughout the developments.

This work has been implemented in the context of the RAWFIE project. RAWFIE has received funding from the European Unions Horizon 2020 Research and Innovation programme under grant agreement No. 645220.

This work is part of the project STRONGMAR: STRengthening MARritime technology Research Center (H2020TWINN-2015 (CSA)-692427).

This work is supported by the ERDF European Regional Development Fund through the Operational Programme for Competitiveness and Internationalisation - COMPETE 2020 Programme within project "POCI-01-0145-FEDER-006961", and by National Funds through the Portuguese funding agency, FCT - Fundao para a Ciłncia e a Tecnologia as part of project “UID/EEA/50014/2013”.

\section{REFERENCES}

[1] Martac, "Mantac - man-portable tactical autonomous system," http://www.martacsystems.com/MANTAS.aspx, April 2017.

[2] K. Kebkal, I. Glushko, T.Tietz, R.Bannasch, O.G.Kebkal, M.Komar, and S.G.Yakovlev, "Sonobot - an autonomous unmanned surface vehicle for hydrographic surveys, hydroacoustic communication and positioning in tasks of underwater acoustic surveillance and monitoring," in Proceedings of the 2nd International Conference and Exhibition on Underwater
Acoustics (UA2014), J. S. Papadakis and L. Bjørnø, Eds., 2014, pp. 1353-1362.

[3] Sonarbeam, "Unmanned surface vehicle," http://www.sonarbeam.co.kr/New/product/eng_ROSB.php, April 2017.

[4] Clearpath, "Clearpath heron," https://www.clearpathrobotics.com/heronbathymetry-unmanned-surface-vessel/, April 2017.

[5] D. Ocean, "Phantom i980," http://www.deepocean.com/usvi-980.php, April 2017.

[6] P. C. Abreu, J. Botelho, P. Góis, A. Pascoal, J. Ribeiro, M. Ribeiro, M. Rufino, L. Sebastião, and H. Silva, "The medusa class of autonomous marine vehicles and their role in eu projects," in OCEANS 2016 Shanghai, April 2016, pp. 1-10.

[7] A. L. Christensen, S. Oliveira, O. Postolache, M. J. De Oliveira, S. Sargento, P. Santana, L. Nunes, F. Velez, P. Sebastiao, V. Costa et al., "Design of communication and control for swarms of aquatic surface drones," in 7th International Conference on Agents and Artificial Intelligence (ICAART). SciTePress, Lisbon, 2015.

[8] N. Cruz, A. Matos, S. Cunha, and S. Silva, "Zarco - an autonomous craft for underwater surveys," in Proceedings of the 7th Geomatic Week, Barcelona, Spain, February 2007., 2007.

[9] B. M. Ferreira, A. C. Matos, and J. C. Alves, "Water-jet Propelled Autonomous Surface Vehicle UCAP: System Description and Control," in OCEANS 2016 - SHANGHAI, ser. OCEANS-IEEE. 345 E 47TH ST, NEW YORK, NY 10017 USA: IEEE, 2016, Proceedings Paper, OCEANS Conference, Shanghai, PEOPLES R CHINA, APR 10-13, 2016.

[10] J. C. Reyes-Guerrero, M. Bruno, L. A. Mariscal, and A. Medouri, "Buoy-to-ship experimental measurements over sea at $5.8 \mathrm{ghz}$ near urban environments," in 2011 11th Mediterranean Microwave Symposium (MMS), Sept 2011, pp. 320-324.

[11] R. G. Garroppo, S. Giordano, and D. Iacono, "Experimental and simulation study of a wimax system in the sea port scenario," in 2009 IEEE International Conference on Communications, June 2009, pp. 1-5.

[12] W. Ejaz, K. Manzoor, H. J. Kim, B. T. Jang, G.J. Jin, and H. S. Kim, "Two-state routing protocol for maritime multi-hop wireless networks," Comput. Electr. Eng., vol. 39, no. 6, pp. 1854-1866, Aug. 2013. [Online]. Available: http://dx.doi.org/10.1016/j.compeleceng.2013.03.011

[13] INESC TEC, "Mare-Fi project," http://win.inesctec.pt/Projects, 2016 (Accessed on 04/11/2017).

[14] L. Santos, "Wi-fi maritime communications using tv white spaces," Master's thesis, University of Porto, Porto, 2013.

[15] M. J. Lopes, F. Teixeira, J. B. Mamede, and R. Campos, "Wi-fi broadband maritime communications using $5.8 \mathrm{ghz}$ band," in 2014 Underwater Communications and Networking (UComms), Sept 2014, pp. $1-5$.

[16] P. Mónica, A. Martins, A. Olivier, A. Matos, J. M. Almeida, N. Cruz, J. C. Alves, H. Salgado, L. Pessoa, P. Jorge, R. Campos, M. Ricardo, C. Pinho, A. Silva, S. Jesus, and E. Silva, "Tec4sea; a modular platform for research, test and validation of technologies supporting a sustainable blue economy," in 2014 Oceans - St. John's, Sept 2014, pp. 1-6.

[17] "Laguna GW2388-4 network processor for wireless embedded designs," http://www.gateworks.com/product/item/laguna-gw2388-4network-processor, (Accessed on 04/11/2017).

[18] "OpenWrt," https://openwrt.org/, (Accessed on 04/11/2017).

[19] "BATMANConcept - Open-Mesh," https://www.openmesh.org/projects/open-mesh/wiki/BATMANConcept, (Accessed on 04/11/2017). 